L'année 2012 a vu le centenaire de la découverte de la diffraction des rayons X par Max von Laue et de la loi de Bragg ; 2013 a vu le centenaire des premières déterminations de structures cristallines. L'Assemblée générale des Nations unies a reconnu l'importance de ces découvertes pour la physique, la chimie, la biologie,

la minéralogie et les sciences de la terre, en déclarant 2014 "Année Internationale de la Cristallographie ».

Les circonstances de

la découverte de la diffraction des rayons $X$ et des premières déterminations de structures cristallines sont évoquées ici.

\title{
Une découverte qui a changé le monde : la diffraction des rayons $X$
}

André Authier (AAuthier@wanadoo.fr)

Institut de Minéralogie et de Physique des Milieux Condensés, Université P. et M. Curie, 4 place Jussieu, 75252 Paris Cedex 05

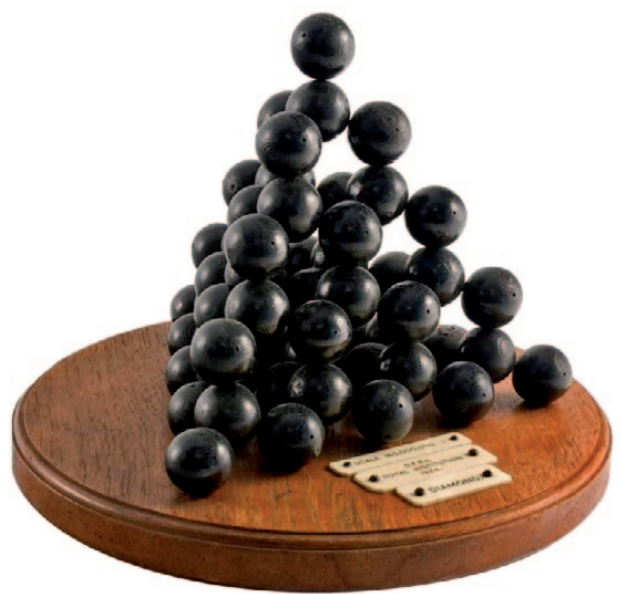

$\mathrm{a}$

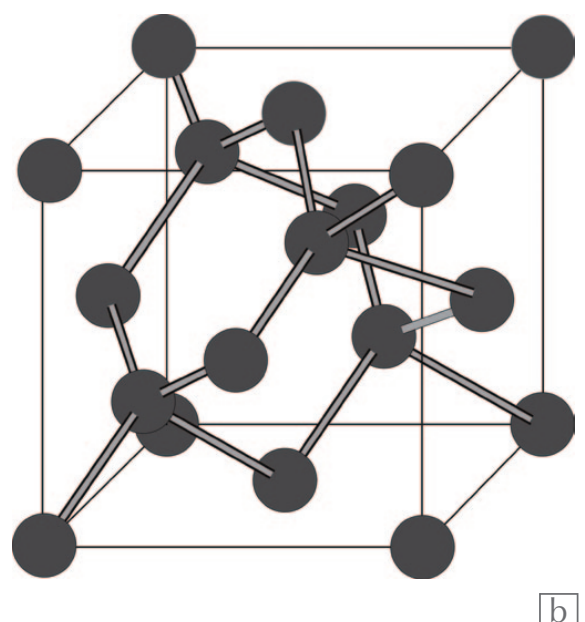

1. Structure cristalline du diamant.

(a) Modèle de W. H. Bragg, montrant la tétravalence des atomes de carbone. Royal Institution, Londres. (Authier 2013).

(b) Maille cubique à faces centrées du diamant.
Chacun sait que les propriétés semiconductrices du silicium, du germanium et de l'arséniure de gallium sont liées à leur structure cristalline de type diamant (fig. 1). Mais quand la structure du diamant a-t-elle été découverte ? La supraconductivité à haute température des cristaux de type $\mathrm{YBaCuO}\left(\mathrm{YBa}_{2} \mathrm{Cu}_{3} \mathrm{O}_{7-\delta}\right)$ découle de leurs propriétés structurales, les propriétés des zéolites sont dues à leur structure en cage, notre ADN a une structure en double hélice. Comment ces structures ont-elles été déterminées ? La plasticité des matériaux est due à la mobilité des dislocations ; a-t-on pu « voir " les dislocations ? Comment l'énergie des niveaux électroniques de l'atome a-t-elle été estimée pour la première fois ? Ces questions peuvent être multipliées à l'infini.

Munich, en 1912, avec l'Institut de Minéralogie de Paul von Groth, l'Institut de Physique Expérimentale de William C. Röntgen et l'Institut de Physique théorique de Arnold Sommerfeld, était le lieu idéal pour la découverte qui allait ouvrir la voie aux réponses à ces questions.

\section{Les rayons $X$ :} ondes ou corpuscules?

Les rayons $\mathrm{X}$ ont été découverts en novembre 1895 par W. C. Röntgen, qui en a aussitôt commencé l'étude des propriétés. Leur nature a été une source de perplexité pour les physiciens. George G. Stokes (1896) et Joseph J. Thomson (1898) en Angleterre, Emil Wiechert (1896) en Allemagne émirent l'hypothèse que ce sont des impulsions électromagnétiques transversales, hypothèse reprise par A. Sommerfeld 
Spectre de diffraction d'une biomolécule. Des images de diffraction de ce type sont produites quand un cristal de protéine est irradié par les rayons $X$ issus d'un accélérateur de particules. Par diffraction de rayons $X$ au HASYLAB/DESY à Hambourg, les scientifiques peuvent décoder la structure interne de molécules complexes de protéines, telles que des enzymes. Source: Max Planck working groups on structural molecular biology, Hamburg.

à Munich qui en a estimé la longueur d'onde moyenne à partir de mesures faites sur des halos de diffraction des rayons $\mathrm{X}$ par une fente (mars 1912).

Charles G. Barkla, en montrant que les rayons $\mathrm{X}$ sont polarisés, en a confirmé ainsi la nature ondulatoire (1905). Mais William Henry Bragg (1907) pensait au contraire qu'il s'agissait de corpuscules qu'il appelait paires neutres, composées d'une particule positive $\alpha$ et d'une particule négative $\beta$.

\section{L'hypothèse des réseaux cristallins}

La régularité et la beauté des cristaux ont été remarquées dès la plus haute antiquité. Ils se présentent sous forme de polyèdres, dont les angles entre faces adjacentes sont indépendants de la provenance de l'échantillon (" loi de constance des angles ", Jean-Baptiste Romé de l'Isle, 1783). La plupart d'entre eux ont, en outre, la propriété de se fendre (cliver) facilement selon certains plans.

C'est l'Abbé René-Just Haüy (1743-1822) qui a jeté en 1784 les bases de la cristallographie moderne, en montrant qu'on ne pouvait expliquer le clivage des cristaux selon des directions spécifiques et la planéité des faces qui composent leurs formes qu'en les supposant constitués par des assemblages tridimensionnels réguliers de petits parallélépipèdes tous identiques, les "molécules intégrantes». Ces empilements de parallélépipèdes constituent les réseaux cristallins (Ludwig Seeber, 1824 ; Gabriel Delafosse, 1848).

Les physiciens français du début du dix-neuvième siècle ont interprété les

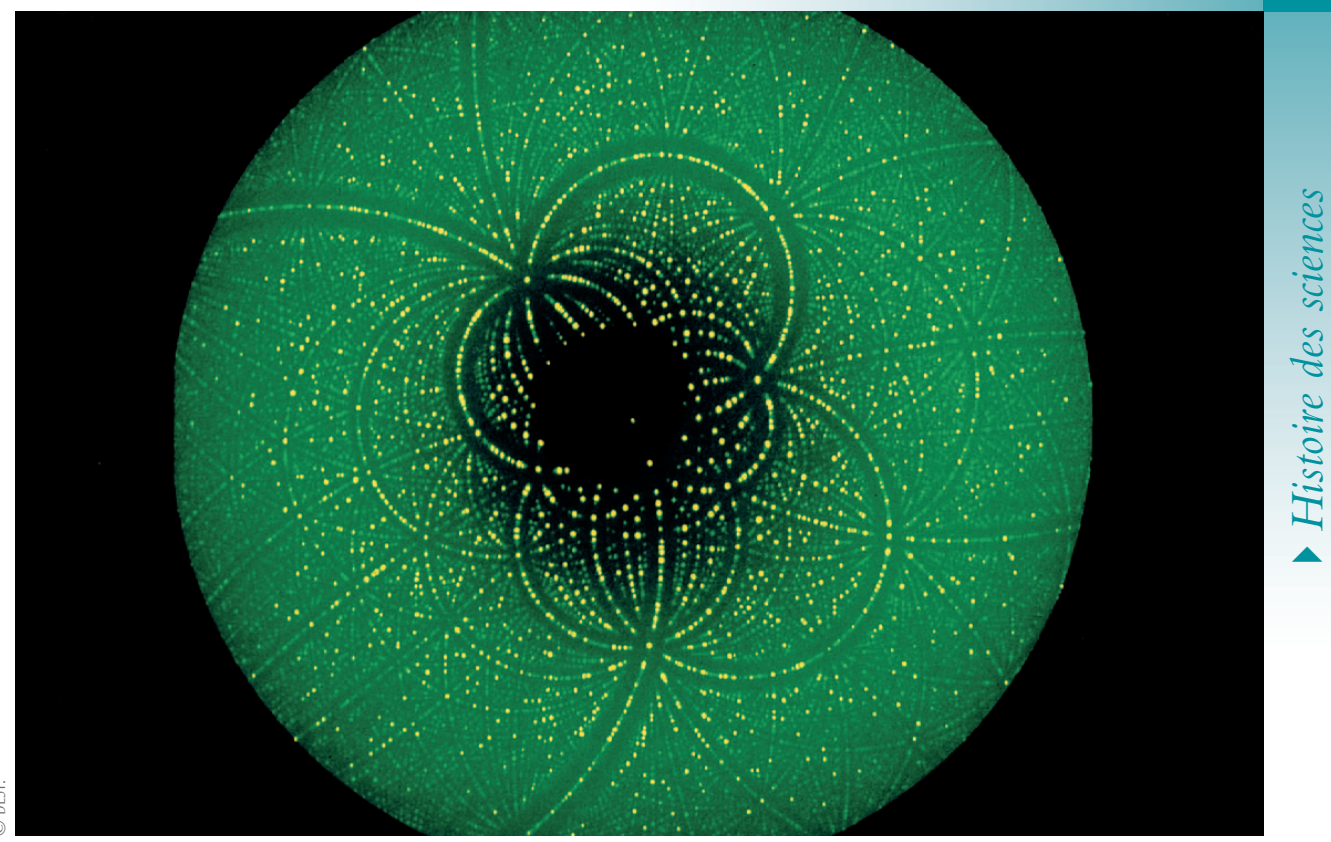

propriétés physiques de la matière solide en la supposant constituée d'une distribution régulière de "molécules ": Fresnel et la double réfraction; Cauchy et les relations entre constantes élastiques.

\section{La thèse d'Ewald}

Au début du vingtième siècle, il n'y avait, en fait, aucune propriété physique que l'on pût relier directement à la structure périodique tridimensionnelle de la matière cristalline. C'est dans cet esprit qu'Arnold Sommerfeld proposa en 1910 à son étudiant Paul P. Ewald de trouver les propriétés optiques d'un ensemble tridimensionnel anisotrope de résonateurs isotropes. Ewald a d'abord étudié la propagation d'une onde dans un tel milieu, supposé infini (théorie de la dispersion), puis la double réfraction d'une onde incidente dans un milieu semi-infini. Cela l'a amené à montrer, au prix de calculs mathématiques ardus (la transformée de Fourier n'était pas encore en usage), que l'onde se propageant dans le milieu cristallin était la somme de deux termes, l'un se propageant à la vitesse de la lumière, $c$, et annihilant l'onde incidente (le théorème de EwaldOseen), l'autre se propageant à la vitesse $c / n$ ( $n$ indice de réfraction) et composée d'une somme infinie d'ondes planes.

Ce calcul était très original pour l'époque et Ewald voulut avoir une confirmation de son raisonnement en montrant son développement en ondes planes à un théoricien réputé, Max Laue, membre comme lui de l'équipe de Sommerfeld (janvier 1912). Laue lui a alors demandé quelle était la distance entre résonateurs.
Ewald ayant répondu qu'elle était de l'ordre de 1/1000 des longueurs d'onde de la lumière, Laue lui demanda si sa théorie et son développement en ondes planes seraient toujours valables si la longueur d'onde des ondes en propagation était elle aussi beaucoup plus courte que celle des ondes lumineuses. La réponse positive d'Ewald a suscité l'intuition de Laue.

\section{L'intuition de Laue}

Max Laue (il n'a signé M. von Laue qu'après l'anoblissement de son père en juin 1913) était à l'époque en train de rédiger, à la demande de Sommerfeld, un article sur l'optique ondulatoire pour une encyclopédie de sciences mathématiques, qui comportait un chapitre sur la diffraction par les réseaux à une et à deux dimensions. L'échange qu'il eut avec Ewald lui donna immédiatement l'idée que si un cristal était un réseau à trois dimensions et que si les rayons X étaient des ondes électromagnétiques, idée prévalant alors à Munich, ils devraient engendrer une figure d'interférence en traversant un cristal. Inspiré par le travail d'Ewald, il pensa, à tort, que les interférences auxquelles il s'attendait seraient dues aux rayons $\mathrm{X}$ émis par fluorescence par les atomes du cristal.

L'idée de Laue fut aussitôt discutée par les physiciens de Munich. Les plus jeunes étaient enthousiastes et Walter Friedrich, un assistant de Sommerfeld qui avait fait sa thèse avec Röntgen, se proposa pour faire l'expérience. Les plus anciens étaient sceptiques, Röntgen parce qu'il avait essayé en vain d'envoyer des rayons $\mathrm{X}$ au travers de lames orientées de cristaux de calcite ou 


\section{>>}

de quartz, Sommerfeld car il craignait que les rayons $\mathrm{X}$ émis par les sources internes au cristal ne soient incohérentes et que les vibrations thermiques ne viennent brouiller les interférences. C'est en effet le moment (mars 1912) où Peter Debye et Max Born commençaient à publier leurs travaux sur la propagation des ondes élastiques dans les cristaux. Mais l'agitation thermique ne fut pas en fait un handicap, à l'étonnement d'Einstein (lettre à Laue du 21 juin 1912).

\section{L'expérience de Friedrich, Knipping et Laue}

Finalement, un doctorant de Röntgen, Paul Knipping, très bon expérimentateur, se joignit à W. Friedrich pour préparer l'expérience suggérée par Laue, un peu dans le dos de leurs patrons respectifs (fig. 2a). Après quelques essais infructueux, le premier cliché montrant des taches d'interférence est obtenu le 21 avril, ainsi qu'en atteste un pli scellé déposé par A. Sommerfeld à l'Académie des Sciences de Bavière le 4 mai 1912. Le premier cristal utilisé était un monocristal de sulfate de cuivre hydraté, $\mathrm{CuSO}_{4}, 5 \mathrm{H}_{2} \mathrm{O}$, choisi pour ses belles formes et parce que le cuivre devait donner, selon Barkla, une forte émission de fluorescence. Les taches obtenues étaient toutefois difficiles à interpréter, par suite du manque de symétrie du cristal (il est triclinique). Friedrich, Knipping et Laue ont alors choisi, sur le conseil de Groth, un cristal de blende, $\mathrm{ZnS}$, cubique et ont alors obtenu des clichés reproduisant la symétrie du cristal (fig. 2b).

L'expérience démontrait ainsi tout à la fois que les rayons $\mathrm{X}$ étaient des ondes et que les cristaux avaient une structure réticulaire. Ce résultat remarquable fut comme un coup de tonnerre et valut à Laue le Prix Nobel de Physique 1914. Les contemporains ne s'y sont pas trompés, comme le minéralogiste Fedorov déclarant que les spectres de diffraction permettaient, en quelque sorte, de voir les atomes avec ses propres yeux (octobre 1912). Laue a donné correctement l'expression mathématique de l'intensité des taches de diffraction, mais s'est trompé dans leur identification sur ses clichés, car il a pensé que dans le sulfure de zinc il y avait des molécules $\mathrm{ZnS}$ aux sommets d'un réseau de cubes.

Mi-juin 1912, Sommerfeld décrivit les résultats de l'expérience à Göttingen.
Parmi l'auditoire se trouvait Ewald, maintenant assistant du mathématicien Felix Klein. Ewald fit instantanément le rapprochement avec son travail de thèse et montra que les taches de diffraction pouvaient s'interpréter très facilement en associant à l'espace physique un espace qu'il appela espace réciproque et que les physiciens ont appelé plus tard espace de Fourier (ou espace des phases), et les mathématiciens espace dual. William Henry Bragg et Arthur Compton montrèrent en effet indépendamment, en 1915, que l'on passait de la distribution électronique des atomes dans l'espace physique à la distribution de l'amplitude diffractée dans l'espace réciproque par une transformée de Fourier.

\section{La loi de Bragg}

Le 26 juin 1912, Laue présenta ses résultats à Würzburg où se trouvait un ancien étudiant de W. H. Bragg, nommé entretemps professeur à Leeds, le norvégien Lars Vegard. Celui-ci s'empressa d'écrire à Bragg pour lui annoncer la nouvelle de cette découverte extraordinaire, qui semblait remettre en cause la théorie corpusculaire des rayons $\mathrm{X}$. Cette nouvelle ébranla effectivement le physicien anglais. Il en discuta longuement pendant l'été 1912 avec son fils Lawrence, alors âgé de 22 ans et étudiant à Cambridge. Ils cherchèrent tout d'abord à expliquer les taches de diffraction par la propagation de corpuscules le long d'avenues entre les rangées d'atomes. Mais l'explication n'était pas satisfaisante et ce fut le fils qui trouva la solution, battant en brèche la théorie de son père.

Se souvenant de la théorie de J. J. Thomson selon laquelle les rayons X étaient des impulsions électromagnétiques, d'une conférence sur les structures cristallines et du cours de Charles T. R. Wilson sur l'optique des réseaux, Lawrence Bragg comprit que les diagrammes de Laue pouvaient être interprétés comme dus à la réflexion d'une onde plane de rayons $\mathrm{X}$ sur des familles de plans réticulaires (novembre 1912). En outre, ces rayons provenaient du spectre continu du tube à rayons $\mathrm{X}$ et non de raies de fluorescence. Adaptant à trois dimensions la loi donnant les positions des maxima d'interférence dans un réseau de traits, il obtint la loi de Bragg (fig. 3). Le professeur de chimie de Cambridge, William Pope, lui ayant dit que le sulfure de zinc avait probablement
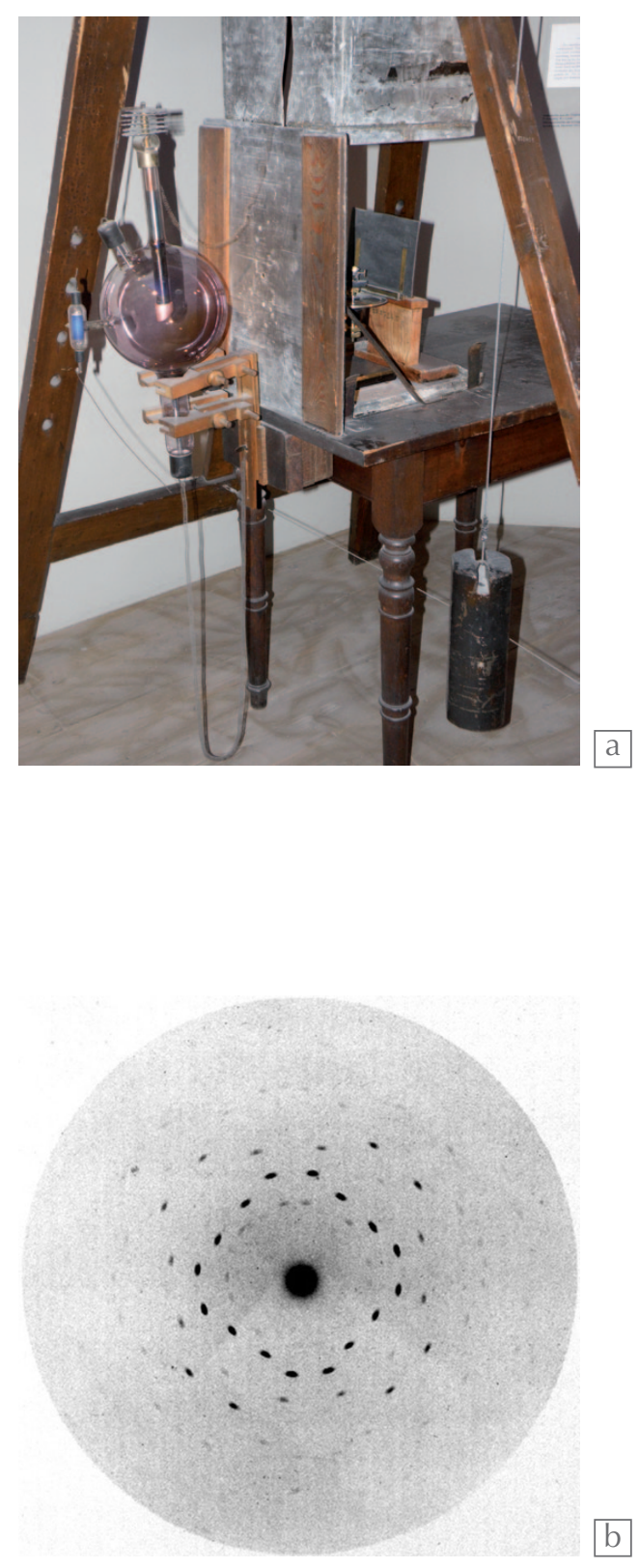

\section{L'expérience de Friedrich, Knipping et Laue.}

(a) Le diffractomètre. Deutsches Museum, Munich. (Authier, 2013).

(b) Diagramme de Laue. Cristal de blende, ZnS, faisceau de rayons $X$ parallèle à un axe de rotation d'ordre 4 , d'après Friedrich, Knipping et Laue (Sitzungsberichte der Kgl. Bayer. Akad. der Wiss. 1912) 


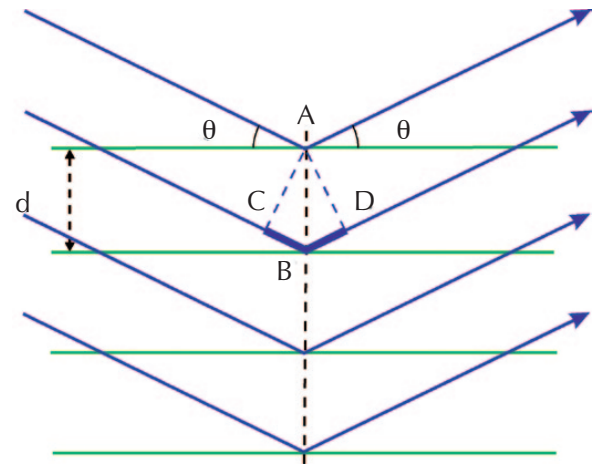

3. Loi de Bragg. Pour que les ondes réfléchies en A et $B$ soient en phase, il faut que leur différence de marche $C B+B D$ soit un nombre entier de longueurs d'onde : $2 d \sin \theta=n \lambda(\lambda$, longueur d'onde ; $d$, équidistance des plans réticulaires; $\theta$, angle entre le faisceau de rayons $X$ et les plans réticulaires).

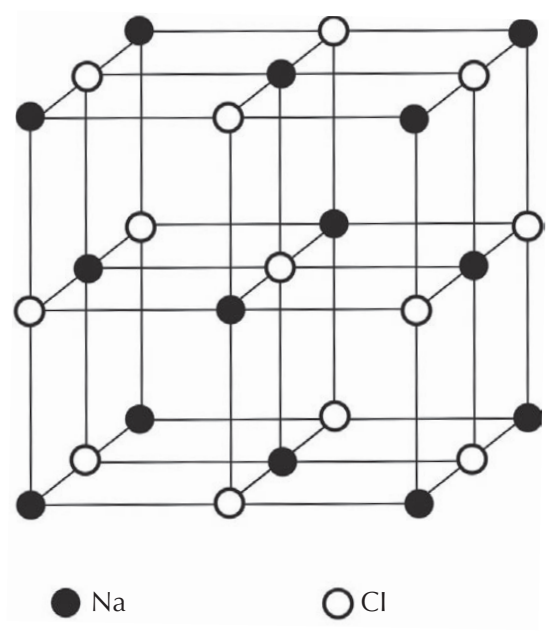

4. Structure cristalline du chlorure de sodium.

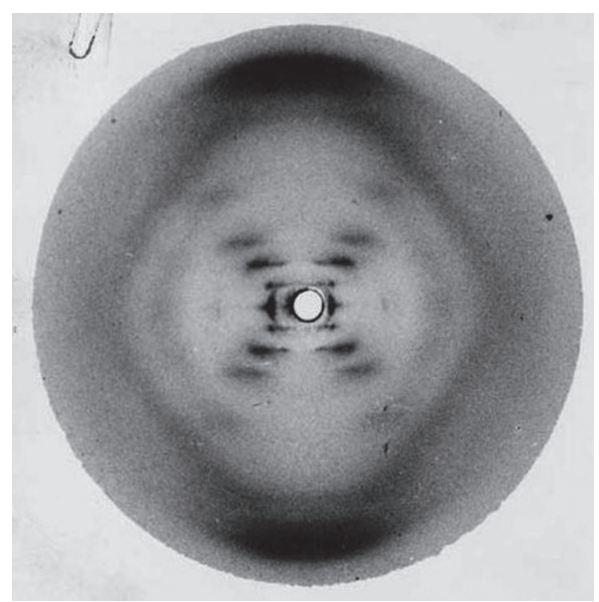

5. Cliché de diffraction de la forme B de l'ADN, obtenu par Rosalind Franklin en 1952 (R.E. Franklin et R.G. Gosling, Nature 1953). Sa forme en X indique la nature hélicoïdale de la molécule de l'ADN. une structure cubique à faces centrées, Lawrence Bragg put interpréter correctement le diagramme de Laue et corriger les erreurs de ce dernier qui avait considéré que le sulfure de zinc avait une structure cubique simple. Pour la première fois, il avait ainsi été possible de déterminer le réseau de Bravais d'un cristal (dans ce cas, cubique à faces centrées) grâce à la diffraction des rayons X. Cela ouvrait la voie à la détermination des structures cristallines.

Le père de Lawrence, William Bragg, admit, un peu à contrecœur, que les rayons $\mathrm{X}$ devaient avoir une double nature, corpusculaire et ondulatoire. L'aspect corpusculaire a été démontré par A. H. Compton avec l'observation de la diffusion inélastique (effet Compton, 1923), et l'interprétation de cette dualité a été fournie par Louis de Broglie à l'aide de la mécanique ondulatoire (1924).

\section{Les premières structures cristallines}

Pendant l'hiver 1912-1913, tandis que Lawrence Bragg prend des clichés, selon la méthode de Laue, de différents halogénures alcalins au Laboratoire Cavendish à Cambridge, son père, à Leeds, monte le premier spectromètre à rayons $\mathrm{X}$, à partir d'un spectromètre optique adapté avec un tube à rayons $\mathrm{X}$ et une chambre d'ionisation, pour enregistrer le diagramme de diffraction.

En combinant les informations obtenues avec les diagrammes de Laue et avec le spectromètre de son père, Lawrence Bragg détermine dès juin 1913 la structure des halogénures alcalins, $\mathrm{NaCl}, \mathrm{KCl}, \mathrm{KBr}$ (fig. 4), montrant qu'un monocristal de sel gemme est une molécule unique, géante, dans laquelle chaque chlore est entouré de six sodium et chaque sodium de six chlore, et non un assemblage de molécules $\mathrm{NaCl}$, ce qui a beaucoup choqué les chimistes de l'époque. Père et fils déterminent ensuite la structure du diamant (juillet 1913), dans laquelle chaque atome de carbone est entouré de quatre atomes de carbone aux sommets d'un tétraèdre (fig. 1), confirmant les idées de Kékulé sur la tétravalence du carbone. Ces résultats eurent une importance considérable pour la chimie. Les déterminations de structures cristallines s'enchaînèrent ensuite. La démonstration que l'on pouvait déterminer la structure atomique de la matière cristalline a été saluée par l'attribution du Prix Nobel de Physique aux Bragg, père et fils, en 1915.
Maurice de Broglie introduisit en 1914 la méthode du cristal tournant pour enregistrer photographiquement le diagramme de diffraction. Indépendamment, Peter Debye et Peter Scherrer en Allemagne $(1915,1916)$ et Alfred Hull aux États-Unis (1916) introduisirent la méthode des poudres. Cette méthode s'est révélée extrêmement féconde, car elle permet d'identifier un échantillon inconnu et d'en déterminer la composition à partir d'une très faible quantité de matière, ce qui est particulièrement utile en métallurgie, en pétrographie et en minéralogie, sans parler des analyses en matière de criminologie. Cette méthode permet aussi d'étudier les contraintes des grosses pièces métalliques et de suivre en temps réel les changements de phase des matériaux.

En 1925, les structures cristallines de plus de 600 composés avaient été déterminées, dont celles de nombreux métaux et alliages et celle du graphite (1924). Alexander Meissner donna en 1927 une première interprétation structurale de la piézoélectricité du quartz (la piézoélectricité a été découverte par les frères Curie en 1880 et la structure cristalline du quartz a été déterminée par W. H. Bragg et Reginald Gibbs en 1925). Katherine Lonsdale, élève de W. H. Bragg, détermina la structure de l'anneau benzénique en 1928. Les structures des principaux silicates furent déterminées entre 1926 et 1930 par l'école de W. L. Bragg, celles des zéolites par Linus Pauling en 1930, qui expliqua leurs propriétés d'échangeurs d'ions par leur structure en cage.

La première structure de macromolécule, celle de la kératine, a été déterminée par l'élève de W. H. Bragg, William Astbury, en 1932. Elle lui permit d'expliquer les propriétés élastiques de la laine. Ce fut le prélude à l'explosion de la biocristallographie : 1949, structure de la pénicilline par Dorothy Hodgkin ; 1950, structure hélicoïdale des protéines (l'hélice $\alpha$ ) par L. Pauling ; 1952, obtention par Rosalind Franklin du fameux cliché de la forme B de l'ADN (fig. 5), dont le dessin en X révélait la structure hélicoïdale et qui fut l'élément déclencheur permettant à James Watson et Francis Crick de découvrir la structure en double hélice de l'ADN (1953). De nos jours, le nombre de structures de protéines déterminées par diffraction des rayons X dépasse 40000 et il n'y a pas un médicament mis sur le marché dont la molécule active ne soit pas le résultat d'une étude par diffraction des rayons $\mathrm{X}$ 
$>>$

(pour les relations structure-fonction des macromolécules, voir Bowler et Nurizzo [1]).

La diffraction des électrons, découverte en 1927, indépendamment par Clinton J. Davisson et Lester H. Germer aux États-Unis et par George P. Thomson et Alexander Reid en Angleterre, et la diffraction des neutrons, découverte par Hans von Halban et Pierre Preiswerk en 1936 à l'aide d'une source radioactive, mais dont le premier praticien a été Cliff Shull en 1946 grâce à un réacteur nucléaire, vinrent compléter la diffraction des rayons $\mathrm{X}$ comme moyens d'investigation de la matière.

C'est précisément par diffraction des électrons que Daniel Schechtman a découvert en 1982 des phases métalliques non périodiques, mais douées de symétrie d'orientation comportant des axes de rotation d'ordre 5, incompatibles avec les réseaux cristallins (D. Gratias [2]). Ces nouveaux composés, appelés quasicristaux, peuvent présenter des phases stables, limitées par des faces planes, ce qui contredit la prévision d'Haüy selon laquelle les faces planes des cristaux impliquent une structure périodique. Dans le cas des quasicristaux, il s'agit d'une structure quasi-périodique.

\section{Spectroscopie aux rayons $X$ et loi de Moseley}

La loi de Bragg, $2 d \sin \theta=n \lambda$, établit une relation entre longueur d'onde des rayons $\mathrm{X}$ diffractés et distance réticulaire du cristal. La réflexion de Bragg d'une radiation de longueur d'onde inconnue, $\lambda$, par un cristal analyseur de paramètres cristallins connus, chlorure de sodium ou calcite en général, permet donc de déduire la valeur de cette longueur d'onde de l'angle de réflexion, $\theta$. Le premier spectre des raies $L$ du platine a été publié par W.H. et W.L. Bragg en avril 1913 (fig. 6) et les premières valeurs de longueurs d'ondes de rayons X par W.H. Bragg en juin 1913, après que W. L. Bragg ait déterminé la structure cristalline du chlorure de sodium et son paramètre réticulaire (juin 1913).

Henry Moseley, un élève du découvreur du noyau de l'atome, Ernest Rutherford, a fait une étude systématique des fréquences des spectres $K$ et $L$ de nombreux éléments et a établi empiriquement en 1913/1914 la loi dite de Moseley, reliant la fréquence $v$ d'une raie au numéro atomique, $Z$ : $v=v_{0} a(Z-b)^{2}$, où $v_{0}$ est la fréquence de Rydberg et $a$ et $b$ des constantes caracté-

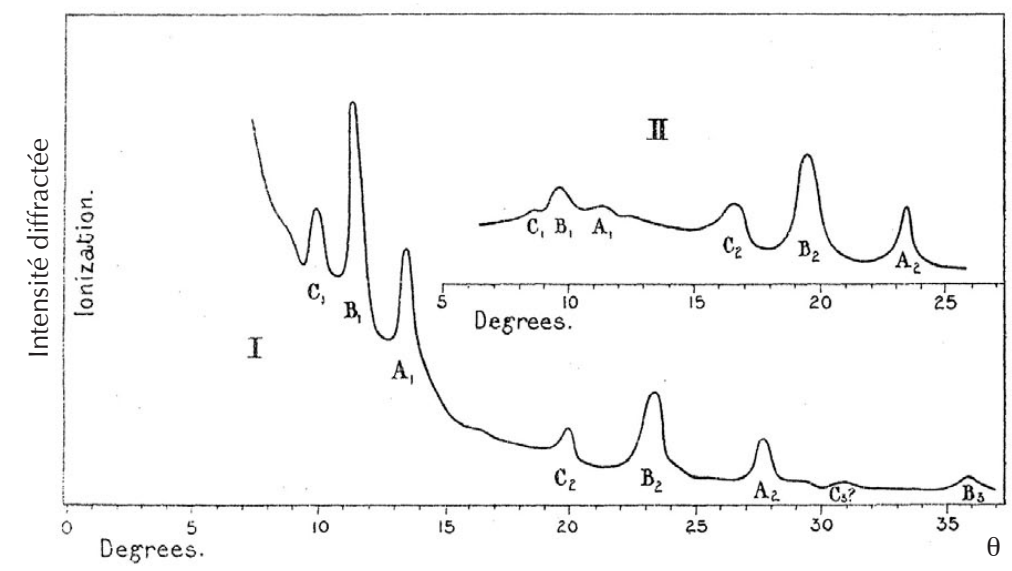

6. Spectre du rayonnement du platine obtenu par réflexion des rayons $X$ émis par une anticathode de platine sur un cristal de chlorure de sodium. Les pics $A, B$ et $C$ correspondent aux raies d'émission $L \alpha$, $L \beta$ et $L \gamma$, respectivement. I : réflexions 200 et 400 par la famille de plans réticulaires (h00) de $\mathrm{NaCl}$; II : réflexions 111 et 222. (D’après W. H. Bragg et W. L. Bragg, Proceedings of the Royal Society A 88 (1913) 428-438.)

ristiques de la raie. C'est par l'étude des spectres d'émission et d'absorption des éléments par diffraction des rayons $\mathrm{X}$ que les niveaux d'énergie des atomes ont été déterminés.

\section{Optique des rayons $X$}

La théorie utilisée pour décrire l'intensité des rayons $\mathrm{X}$ diffractés par les cristaux lors des déterminations de structures cristallines est une théorie simplifiée, dite théorie géométrique ou cinématique, introduite par M. von Laue (1912) et par C. Darwin (1914). Elle ne s'applique qu'à de petits cristaux ou à des cristaux très imparfaits. Pour décrire la diffraction par un cristal parfait, il faut utiliser une théorie plus élaborée, dite théorie dynamique, développée indépendamment par C. Darwin (1914) et par P. P. Ewald (1917). C. Darwin a montré qu'une onde plane incidente sur une face cristalline orientée selon l'angle de Bragg subit une réflexion totale. Cette importante propriété est utilisée dans les monochromateurs et les systèmes optiques pour le rayonnement synchrotron.

Selon la théorie d'Ewald, dérivée de sa thèse et modifiée par M. von Laue (1931), les rayons $\mathrm{X}$ se propagent dans les cristaux sous forme d'associations d'ondes planes, physiquement liées, appelées champs d'ondes, analogues aux ondes de Bloch en physique du solide. Une onde plane incidente sur un cristal génère deux champs d'ondes, dont l'un est absorbé de manière anormalement faible, et l'autre de manière anormalement forte, phénomène observé par G. Borrmann (1941) et calculé par M. von Laue (1949). Leurs directions de propagation dans le cristal sont différentes (Laue 1952) et il y a donc double réfraction de l'onde incidente dans le cristal, prévue par G. Borrmann (1955) et observée par Authier (1960).

La propagation des champs d'onde dans le cristal est perturbée par les champs de contraintes dues aux défauts étendus tels que les dislocations, ce qui permet d'obtenir une image de la position de ces défauts dans le cristal (topographie aux rayons X, inventée par Lang et par Borrmann en 1958) et de "voir » ainsi les dislocations.

\section{Conclusion}

La diffraction des rayons $\mathrm{X}$, des électrons et des neutrons a complètement bouleversé nos moyens d'observation de la structure de la matière, à l'échelon atomique ou moléculaire ainsi qu'à l'échelle intra-atomique. Leur impact se fait sentir dans tous les domaines du monde moderne : science des matériaux avec les semi-conducteurs et les supraconducteurs, les superalliages et les superaimants, nanotechnologie, biochimie et biotechnologie avec l'étude des protéines de la vie et la conception de nouveaux médicaments. Cet impact a été reconnu par l'attribution de plus d'une vingtaine de Prix Nobel, le dernier en 2013, sur la modélisation de réactions chimiques entre macromolécules dont la structure a été déterminée par diffraction des rayons $\mathrm{X}$. 


\section{Les découvreurs de la diffraction des rayons $\mathrm{X}$}

\section{Max von Laue}

Né le 9 octobre 1879 à Pfaffendorf, Allemagne, mort le 24 avril 1960 à Berlin, Allemagne. Après des études à Strasbourg et Göttingen, il prépare sa thèse à Berlin sous la direction de M. Planck, soutenue en 1905. Il est nommé Privatdozent à Munich, puis, successivement, Professeur à Zürich en 1913, à Francfort en 1914, puis enfin à Berlin en 1919. II reçoit le prix Nobel de Physique en 1914.
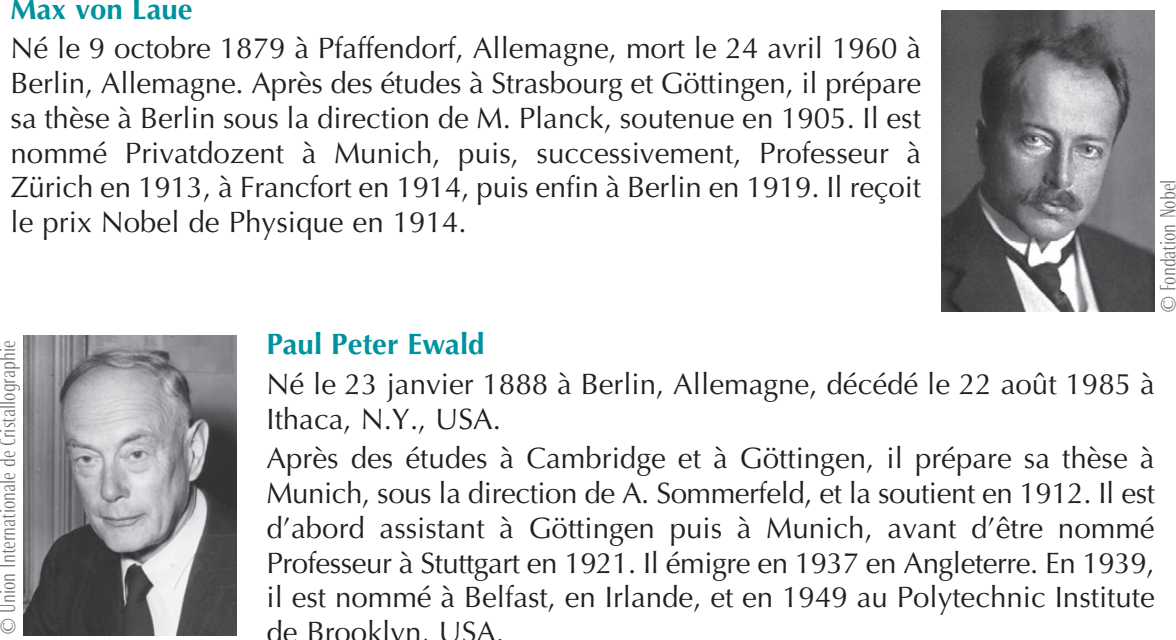

Paul Peter Ewald

Né le 23 janvier 1888 à Berlin, Allemagne, décédé le 22 août 1985 à Ithaca, N.Y., USA.

Après des études à Cambridge et à Göttingen, il prépare sa thèse à Munich, sous la direction de A. Sommerfeld, et la soutient en 1912. Il est d'abord assistant à Göttingen puis à Munich, avant d'être nommé Professeur à Stuttgart en 1921. Il émigre en 1937 en Angleterre. En 1939, il est nommé à Belfast, en Irlande, et en 1949 au Polytechnic Institute de Brooklyn, USA.

William Henry Bragg

Né le 2 juillet 1862 à Wigton, Angleterre, décédé le 21 mars 1942 à Londres, Angleterre. Après des études à Cambridge, il est nommé Professeur de Physique à Adelaide en Australie. II revient en Angleterre en 1909 comme Professeur à l'Université de Leeds. En 1915 il est nommé à University College, à Londres, mais ne prend ses fonctions qu'à la fin de la guerre. En 1923, il est nommé Professeur à la Royal Institution, à Londres. En 1915, il reçoit le prix Nobel de Physique, conjointement avec son fils.
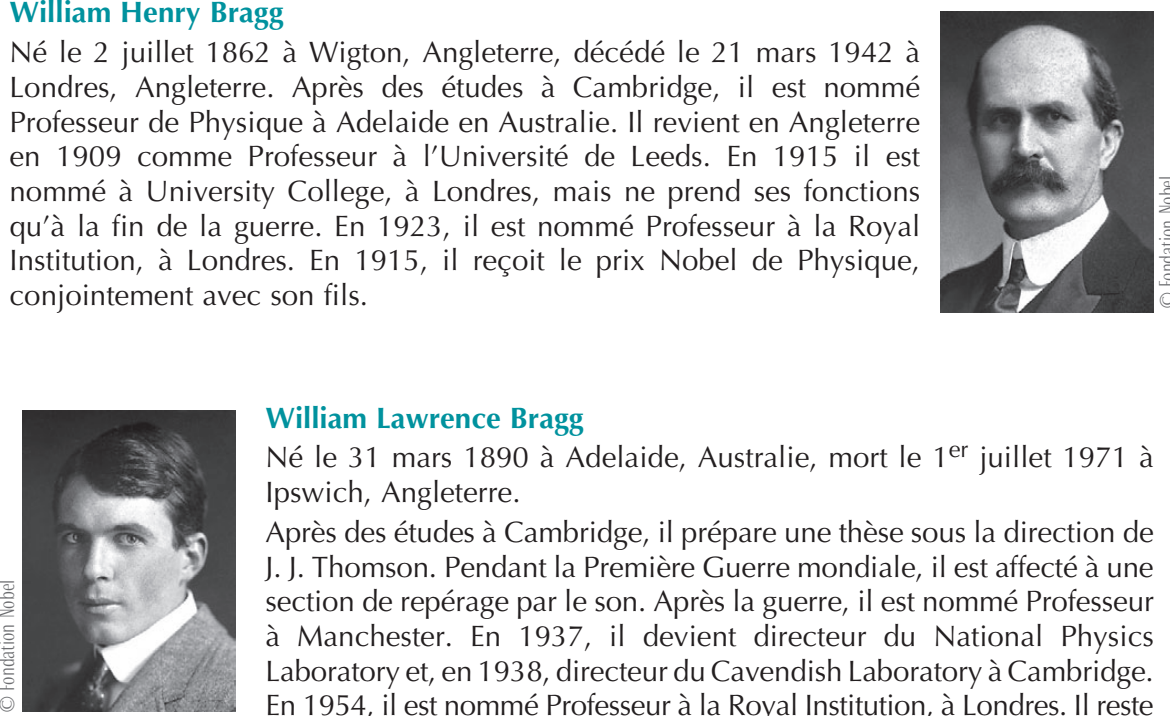

William Lawrence Bragg

Né le 31 mars 1890 à Adelaide, Australie, mort le $1^{\text {er }}$ juillet 1971 à Ipswich, Angleterre.

Après des études à Cambridge, il prépare une thèse sous la direction de J. J. Thomson. Pendant la Première Guerre mondiale, il est affecté à une section de repérage par le son. Après la guerre, il est nommé Professeur à Manchester. En 1937, il devient directeur du National Physics Laboratory et, en 1938, directeur du Cavendish Laboratory à Cambridge. En 1954, il est nommé Professeur à la Royal Institution, à Londres. II reste le plus jeune récipiendaire du Prix Nobel, reçu avec son père en 1915.

\section{Références}

- A. Authier, Early days of X-ray diffraction, 0xford University Press (2013).

- P. P. Ewald, Fifty years of X-ray diffraction, published for the International Union of Crystallography,

N V. A. Oosthoek, Utrecht (1962).

www.iucr.org/publ/50yearsofxraydiffraction/full-text

- J. R. Helliwell et al., "Some historical extracts relevant to the discovery and application of the diffraction of $X$-rays by crystals to contribute to the Centennial celebration and the International Year of

Crystallography", Crystallography Reviews, 18 (2012) 3-19.
-J.-L. Hodeau et R. Guinebretière, “Crystallography: past and present”, Appl. Phys. A, 89 (2007) 813-823.

- C. Kaspar et C. Jaussaud, « Physique et Minéralogie au Museum », Bulletin de la SFP 154 (2006) 21-24.

1• M.W. Bowler et D. Nurizzo, « Le rayonnement synchrotron : comprendre les relations structure-fonction des macromolécules biologiques ", Reflets de la physique 34-35 (2013) 48-53.

2 D. Gratias, "Les quasicristaux ", Reflets de la physique, 29 (2012) 12-16. 